\title{
Individual rationality and voting in cooperative production
}

\author{
Luis C. Corchón $^{\mathrm{a}, *}$, M. Socorro Puy ${ }^{\mathrm{b}}$ \\ ${ }^{a}$ Department of Economics, Universitat Pompeu Fabra, Barcelona 08005, Spain \\ ${ }^{\mathrm{b}}$ Departamento de Fundamentos del Análisis Económico, Universidad de Alicante, Alicante 03071, Spain
}

Received 26 September 1997; accepted 23 January 1998

\begin{abstract}
Suppose that a group of individuals owns collectively a technology which produces a consumption good from an input. A sharing rule associates input contributions with a vector of consumption. We consider sharing rules that are a convex combination of the Proportional, the Equal Share and the Equal Benefit Rules. We characterize the subset of sharing rules that satisfy Pareto efficiency and individual rationality. We also study the outcome of majority voting on this subselection of sharing rules. (C) 1998 Elsevier Science S.A.
\end{abstract}

Keywords: Sharing rule; Individual rationality; Voting

JEL classification: D50; D51; L31; L32; H82; P13

\section{Introduction}

Consider a cooperative, i.e. a group of people owning a technology which transforms an input (labor) in an output (consumption). Inputs are provided by owners. Different proposals on how to distribute the output can be found in the literature. Roemer and Silvestre (1989), (1993) proposed the Proportional Solution and the Equal Benefit Solution and Mas-Colell (1980) proposed the Constant Returns Equivalent Solution (see Roemer (1996) for a survey of this literature). Characterizations of these and other solutions are provided in Moulin (1990); Moulin and Roemer (1989); Maniquet (1996).

In a previous paper (Corchón and Puy, 1997) we present a concept that brings together most of the previous contributions. It is that of a sharing rule. A sharing rule is a function which specifies the list of consumptions as a function of input contributions. All solutions mentioned above are sharing rules except the constant returns equivalent solution. We found that all continuous sharing rules are compatible with efficiency (i.e. there are allocations which belong to the sharing rule and are Pareto efficient) and are Nash implementable. We conclude that neither efficiency nor incentives have any cutting power to reduce the large set of continuous sharing rules.

In this paper we propose alternative properties in order to select efficient sharing rules in economies with an homogeneous input. This subset, referred to in the sequel as the admissible set, consists of the

\footnotetext{
*Corresponding author. Tel.: (34-9) 35421672; fax: (34-9) 35421746; e-mail: luis.corchon@econ.upf.es
} 
convex combination of the Equal Share, the Proportional and the Equal Benefit sharing rules. These rules are chosen for two reasons: analytical tractability and popularity in the literature. We investigate the consequences of imposing two requirements: individual rationality (i.e. there are allocations which belong to the sharing rule that are Pareto efficient and give, at least, the reservation utility to all agents) and majority voting among individually rational sharing rules. Our first result, recorded as Proposition 1, is that a necessary and sufficient condition for a sharing rule in the admissible set to be individually rational is that the weight given to the equal share rule must be zero. Then, we restrict attention to economies with quasilinear preferences in the consumption good. This assumption is equivalent to say that the efficient allocation of labor is independent of consumption, which could be a good approximation in situations where consumption is not very low. In this class of economies we show that if the number of agents working more (resp. less) than the average is larger than half of the whole population, the outcome of majority voting is the Proportional rule (resp. Equal Benefit rule) (Proposition 2).

\section{The model}

There is one consumption good produced from a vector of homogeneous inputs using a publicly owned technology.

There are $n$ individuals indexed by $i$. Let $N=\{1, \ldots, n\}$. They are endowed each with $\bar{\ell}_{i} \in \mathbb{R}_{+}$units of input. Each individual consumption set is defined by

$$
\mathbb{X}_{i}=\left\{\left(x_{i}, \ell_{i}\right): x_{i} \in \mathbb{R}_{+}, \ell_{i} \in\left[0, \bar{\ell}_{i}\right]\right\}
$$

where $x_{i}$ is agent $i$ 's consumption and $\ell_{i}$ is her input contribution. Each agent has preferences defined on $\mathbb{X}_{i}$, which can be represented by a utility function:

$$
u_{i}: \mathbb{X}_{i} \rightarrow \mathbb{R}
$$

This function is assumed to be concave, differentiable, increasing in $x_{i}$ and decreasing in $\ell_{i}$. Let $\vartheta_{i}$ be the space of all utility functions satisfying these assumptions. The technology is represented by a production function

$$
f: \mathbb{R}_{+} \rightarrow \mathbb{R}
$$

The argument of $f$ is $\Sigma_{i \in N} \ell_{i}$, and $f$ is increasing, concave, differentiable and $f(0)=0$. Let $\mathscr{F}$ be the space of technologies satisfying these assumptions.We define the feasible set, denoted by $\mathbb{X}$ as follows:

$$
\mathbb{X}=\left\{\left(x_{1}, \ell_{1}, \ldots, x_{n}, \ell_{n}\right) \in \prod_{i \in N} \mathbb{X}_{i}: \sum_{i \in N} x_{i} \leq f\left(\sum_{i \in N} \ell_{i}\right)\right\}
$$

A feasible allocation is denoted by $(x, \ell) \in \mathbb{X}$ where $x=\left(x_{1}, \ldots, x_{n}\right)$ and $\ell=\left(\ell_{1}, \ldots, \ell_{n}\right)$. An economy is denoted by $e=\{u, f\}$, where $u=\left(u_{1}, \ldots, u_{n}\right)$ is a list of utility functions. The set of economies is denoted by $\mathscr{E}=\prod_{i \in N} \mathcal{U}_{i} \times \mathscr{F}$. The Pareto efficient solution $\varphi^{E}: \mathscr{E} \rightarrow \mathbb{X}$ associates to each economy in the domain the set of Pareto efficient allocations for this economy. Formally, 


$$
\varphi^{E}(u, f)=\left\{\begin{array}{c}
(x, \ell) \in \mathbb{X}: \exists\left(x^{\prime}, \ell^{\prime}\right) \in \mathbb{X} / u_{h}\left(x_{h}^{\prime}, \ell_{h}^{\prime}\right) \geq u_{h}\left(x_{h}, \ell_{h}\right) h \in N \\
\text { and } u_{j}\left(x_{j}^{\prime}, \ell_{j}^{\prime}\right)>u_{j}\left(x_{j}, \ell_{j}\right) \text { for at least one } j \in N
\end{array}\right\}
$$

A sharing rule $P=\left(P_{1}, \ldots, P_{n}\right)$ is a list of functions such that

$$
P_{i}: \prod_{i \in N}\left[0, \bar{\ell}_{i}\right] \times \mathscr{F} \rightarrow \mathbb{R}_{+} \forall i \in N \text { and } \sum_{i \in N} P_{i}(\ell, f)=f\left(\sum_{i \in N} \ell_{i}\right) \forall \ell \in \prod_{i \in N}\left[0, \bar{\ell}_{i}\right]
$$

Each $P_{i}$ yields the consumption of $i$ as a function of $\ell$ and $f$. Moreover $P$ distributes the whole output. When the context is clear we will write $P_{i}(\ell)$. In particular, we define the following three sharing rules.

The Proportional Rule:

$$
P_{i}^{p}(\ell)=\frac{f\left(\sum_{i \in N} \ell_{i}\right)}{\sum_{i \in N} \ell_{i}} \ell_{i} \forall i \in N,
$$

in which the amount of output consumed by an agent is proportional to the amount of input that she contributes.

The Equal Benefit rule:

$$
P_{i}^{b}(\ell)=\frac{f\left(\sum_{i \in N} \ell_{i}\right)}{n}+\frac{\partial f\left(\sum_{i \in N} \ell_{i}\right)}{\partial \ell_{i}}\left(\ell_{i}-\frac{\sum_{i \in N} \ell_{i}}{n}\right) \forall i \in N,
$$

in which each agent consumes according to her budget constraint of the Walrasian equilibrium with equal distribution of profits.

The Equal Share rule:

$$
P_{i}^{s}(\ell)=\frac{f\left(\sum_{i \in N} \ell_{i}\right)}{n} \forall i \in N,
$$

in which each agent consumes an equal part of the total output. In this note, we focus attention on a subfamily of sharing rules denoted by $\mathscr{P}$ that consists of the convex combinations of the three sharing rules defined above (see Moulin, 1987; Pfingsten, 1991 for similar examples). We denote a typical element of this subfamily by $\bar{P}=\left(\bar{P}_{1}, \ldots, \bar{P}_{n}\right)$. Thus,

$$
\bar{P}_{i}(\ell)=(\alpha+\gamma) \frac{f\left(\sum_{i \in N} \ell_{i}\right)}{n}+\beta \frac{f\left(\sum_{i \in N} \ell_{i}\right)}{\sum_{i \in N} \ell_{i}} \ell_{i}+\gamma \frac{\partial f\left(\sum_{i \in N} \ell_{i}\right)}{\partial \ell_{i}}\left(\ell_{i}-\frac{\sum_{i \in N} \ell_{i}}{n}\right)
$$

where $\alpha, \beta, \gamma \in \mathbb{R}_{+}$and by adding up over $i$ we get that $\alpha+\beta+\gamma=1$. In the sequel we consider only 
interior allocations $(x, \ell)$ satisfying $x_{i}=\bar{P}_{i}(\ell) \forall i \in N$ and Pareto efficiency. These allocations are denoted by $\varphi^{S E}(u, f, \bar{P})$,

$$
\varphi^{S E}(u, f, \bar{P})=\left\{(x, \ell) \in \varphi^{E}(u, f): x_{i}=\bar{P}_{i}(\ell) \forall i \in N\right\} .
$$

Notice that every $\bar{P}$ is the sum of continuous sharing rules. Therefore Theorem 1 in Corchón and Puy (1997) implies that for every $\bar{P} \in \mathscr{P}, \varphi^{S E}(u, f, \bar{P}) \neq \phi$.

\section{Individual rationality of sharing rules}

First, let us define individual rationality in our framework.

Definition. We say that a sharing rule $P$ is efficient and individually rational if for every $(u, f) \in \mathscr{E}$ there is an allocation $(x, \ell) \in \varphi^{S E}(u, f, P)$ such that $u_{i}\left(x_{i}, \ell_{i}\right) \geq u_{i}(0,0) \forall i \in N$.

In the above definition we assume that the outside option is zero consumption and zero labor contribution. Other assumptions on the outside option are also possible (see Fleurbaey and Maniquet, 1996, p. 204). The one used here is the most basic since no work and no consumption is always possible.

In this section we characterize for the set of all classical economies the subset of sharing rules contained in $\mathscr{P}$ that verify efficiency and individual rationality. The key condition that characterizes this subset is the following property:

Definition. A sharing rule P verifies Proportionality on Linear Economies (PLE) if P coincides with the Proportional Rule when the technology $f$ displays constant returns to scale.

It is easily seen that PLE is equivalent to the axiom of Free Access on Linear Economies introduced by Roemer and Silvestre (1989).

Lemma 1: For every $\bar{P} \in \mathscr{P}, \alpha=0$ if and only if PLE holds.

Proof. Let us prove necessity. Under constant returns to scale

$$
f^{\prime}(\ell)=\frac{f\left(\sum_{i \in N} \ell_{i}\right)}{\sum_{i \in N} \ell_{i}},
$$

and thus,

$$
\bar{P}_{i}(\ell)=f\left(\sum_{i \in N} \ell_{i}\right)\left[\alpha \frac{1}{n}+(\beta+\gamma) \frac{\ell_{i}}{\sum_{i \in N} \ell_{i}}\right] \forall i \in N
$$

PLE implies that, 


$$
f\left(\sum_{i \in N} \ell_{i}\right)\left[\alpha \frac{1}{n}+(\beta+\gamma) \frac{\ell_{i}}{\sum_{i \in N} \ell_{i}}\right] \equiv \frac{f\left(\sum_{i \in N} \ell_{i}\right)}{\sum_{i \in N} \ell_{i}} \ell_{i}
$$

and substituting that $\alpha+\beta+\gamma=1$ we obtain:

$$
\alpha \frac{1}{n} \equiv \alpha \frac{\ell_{i}}{\sum_{i \in N} \ell_{i}}
$$

But this equation must hold for every $\ell$ and thus, $\alpha=0$. Sufficiency is obvious. Q.E.D.

Proposition 1: For every $(u, f) \in \mathscr{E}, P L E$ is a necessary and sufficient condition for $\bar{P} \in \mathscr{P}$ to be an efficient and individually rational sharing rule.

Proof. First we show that PLE is a necessary condition. Assume that $\bar{P} \in \mathscr{P}$ but it does not verify PLE. Then we will show that there is an economy $(\bar{u}, \bar{f}) \in \mathscr{E}$ with two agents and where $\bar{f}$ displays constant returns to scale, such that $\bar{P}$ is not efficient and individually rational.

Because $\bar{P}$ does not verify PLE, we find that there is an allocation $\left(x^{*}, \ell^{*}\right)$ such that there is a $i$ for whom $\bar{P}_{i}\left(\ell^{*}\right)<P_{i}{ }^{p}\left(\ell^{*}\right)$. W.l.o.g. take $i=1$.

Let $(\bar{u}, \bar{f})$ be such that $\bar{u}_{1}=x_{1}-\left(s \ell_{1}^{a+1} / a+1\right), \bar{u}_{2}=x_{2}-t \ell_{2}^{2}$ with $a \in \mathscr{R}_{+}, \bar{f}(\ell)=\Sigma_{i \in N} \ell_{i}, t=(1 /$ $\left.2 \ell_{2}^{*}\right)$ and $s=\left(1 / \ell_{1}^{* a}\right)$. We find that $\left(\bar{P}\left(\ell^{*}\right), \ell^{*}\right)=\varphi^{S E}(\bar{u}, \bar{f}, \bar{P})$ for every $a$, i.e. $\varphi^{S E}(\bar{u}, \bar{f}, \bar{P})$ contains a unique allocation. But because $\bar{P}_{1}\left(\ell^{*}\right)<\ell_{1}^{*}=P_{1}{ }^{p}\left(\ell^{*}\right)$, taking $a$ sufficiently close to $0, \bar{u}_{1}=\bar{P}_{1}\left(\ell^{*}\right)-$ $\left(\ell_{1}^{*} /(1+a)\right) \simeq \bar{P}_{1}\left(\ell^{*}\right)-\ell_{1}^{*}<0$ which violates individual rationality. Thus, $\bar{P}$ is not efficient and individually rational.

Second we show that PLE is a sufficient condition.

Let us define the function $\phi_{i}{ }^{0}\left(\ell_{i}\right)=x_{i}$ as the indifference curve of agent $i$, that passes through the origin $\left(x_{i}, \ell_{i}\right)=(0,0)$. By concavity of $u_{i}, \phi_{i}{ }^{0}$ is a convex function and thus:

$$
\frac{\partial \phi_{i}^{0}\left(\ell_{i}\right)}{\partial \ell_{i}} \geq \frac{\phi_{i}^{0}\left(\ell_{i}\right)}{\ell_{i}} \text { for } \forall i \in N,
$$

from the implicit function theorem we have that

$$
\frac{\partial \phi_{i}^{0}\left(\ell_{i}\right)}{\partial \ell_{i}}=-\frac{\frac{\partial u_{i}\left(x_{i}, \ell_{i}\right)}{\partial \ell_{i}}}{\frac{\partial u_{i}\left(x_{i}, \ell_{i}\right)}{\partial x_{i}}}
$$

Next, let us assume that the result is not true. This implies that there is an economy $(u, f) \in \mathscr{E}$ and a $P^{\prime} \in \mathscr{P}$ satisfying PLE such that for every $\left(x^{\prime}, \ell^{\prime}\right) \in \varphi^{S E}\left(u, f, P^{\prime}\right)$,

$$
P_{i}^{\prime}\left(\ell^{\prime}\right)=x_{i}^{\prime}<\phi_{i}^{0}\left(\ell_{i}^{\prime}\right) \text { for at least one } i \in N,
$$


By Pareto efficiency and interiority of $\left(x^{\prime}, \ell^{\prime}\right)$, the following condition holds:

$$
\frac{\frac{\partial u_{i}\left(x_{i}^{\prime}, \ell_{i}^{\prime}\right)}{\partial x_{i}}}{\frac{\partial u_{i}\left(x_{i}^{\prime}, \ell_{i}^{\prime}\right)}{\partial \ell_{i}}}=\frac{-1}{\frac{\partial f\left(\sum_{i \in N} \ell_{i}^{\prime}\right)}{\partial \ell_{i}}} \forall i \in N .
$$

From Eqs. (3.1)-(3.4) we have that

$$
P_{i}^{\prime}\left(\ell^{\prime}\right)<\phi_{i}^{0}\left(\ell_{i}^{\prime}\right) \leq \ell_{i}^{\prime} \frac{\partial \phi_{i}^{0}\left(\ell_{i}^{\prime}\right)}{\partial \ell_{i}}=\ell_{i}^{\prime} \frac{\partial f\left(\sum_{i \in N} \ell_{i}^{\prime}\right)}{\partial \ell_{i}} \text { for at least one } i \in N .
$$

Substituting $P_{i}^{\prime}$ and by concavity of $f$,

$$
\frac{\partial f\left(\sum_{i \in N} \ell_{i}^{\prime}\right)}{\partial \ell_{i}}\left[(\alpha+\gamma) \frac{\sum_{i \in N} \ell_{i}^{\prime}}{n}+\beta \ell_{i}^{\prime}+\gamma\left(\ell_{i}^{\prime}-\frac{\sum_{i \in N} \ell_{i}^{\prime}}{n}\right)\right]<\ell_{i}^{\prime} \frac{\partial f\left(\sum_{i \in N} \ell_{i}^{\prime}\right)}{\partial \ell_{i}} .
$$

Simplifying and taking into account that $\beta=1-\alpha-\gamma$, we obtain that

$$
\alpha \frac{\sum_{i \in N} \ell_{i}^{\prime}}{n}<\alpha \ell_{i}^{\prime}
$$

Finally, by PLE and Lemma 1, we have that $\alpha=0$; but then we obtain a contradiction. Q.E.D. Notice that the necessity part works for any set of admissible sharing rules.

\section{Voting on sharing rules}

In this section we consider the subset of sharing rules that verify efficiency and individual rationality in the set $\mathscr{P}$. By the result obtained in Section 3 these sharing rules can be expressed as follows for each $i \in N$,

$$
\hat{P}_{i}(\gamma, \ell)=\gamma\left(\frac{f\left(\sum_{i \in N} \ell_{i}\right)}{n}+\frac{\partial f\left(\sum_{i \in N} \ell_{i}\right)}{\partial \ell}\left(\ell_{i}-\frac{\sum_{i \in N} \ell_{i}}{n}\right)\right)+(1-\gamma) \frac{f\left(\sum_{i \in N} \ell_{i}\right)}{\sum_{i \in N} \ell_{i}} \ell_{i} .
$$

To facilitate the analysis we assume that $n$ is an odd number. In the sequel we assume that the preferences of the individuals are represented by quasilinear utility functions, i.e.

$$
u_{i}\left(x_{i}, \ell_{i}\right)=x_{i}-v_{i}\left(\ell_{i}\right), \forall i \in N,
$$


where $v_{i}$ is a convex and increasing function. Let $\mathscr{E}^{\prime}=(u, f)$ be the set of economies where $u$ is a profile of utility functions satisfying the previous assumption and $f$ is a strictly concave production function. In this set of economies each $\gamma$ not only specifies a sharing rule, but it also determines uniquely an efficient allocation $(x, \ell) \in \varphi^{S E}(u, f, \hat{P})$ (as is made clear in the proof below).

Lemma 2: For every economy $(u, f) \in \mathscr{E}$, each agent preferences defined over $\gamma \in[0,1]$ are single peaked with peak in $\gamma=0$ or $\gamma=1$.

Proof. Take an economy $(u, f) \in \mathscr{E}^{\prime}$. We first show that each $\gamma \in[0,1]$ determines a unique allocation $(\hat{x}, \hat{\ell}) \in \varphi^{S E}(u, f, \hat{P}(\gamma, \ell))$. By Pareto efficiency we find that the allocation $(\hat{x}, \hat{\ell})$ satisfies the condition:

$$
\frac{\partial f\left(\sum_{i \in N} \ell_{i}\right)}{\partial \ell_{i}}=\frac{\partial v_{i}\left(\ell_{i}\right)}{\partial \ell_{i}} \text { for } \forall i \in N .
$$

By strict convexity of preferences, the above condition determines a unique vector $\hat{\ell}$ of input contributions that is independent of $\gamma$.

Therefore the first derivative of $u_{i}$ with respect to $\gamma \forall i \in N$, is:

$$
\frac{\partial u_{i}\left(\hat{P}(\gamma, \hat{\ell}), \hat{\ell}_{i}\right)}{\partial \gamma}=\frac{\partial \hat{P}(\gamma, \hat{\ell})}{\partial \gamma}=\left(\frac{f\left(\sum_{i \in N} \hat{\ell}_{i}\right)}{\sum_{i \in N} \hat{\ell}_{i}}-\frac{\partial f\left(\sum_{i \in N} \ell_{i}\right)}{\partial \ell}\right) \cdot\left(\frac{\sum_{i \in N} \hat{\ell}_{i}}{n}-\hat{\ell}_{i}\right)
$$

By strict concavity of $f$, the first term of the expression is always positive. We then find two kinds of agents:Those agents for whom $\left(\sum_{i \in N} \hat{\ell}_{i}\right) /(n)>\hat{\ell}_{i}$ and those agents for whom $\left(\sum_{i \in N} \hat{\ell}_{i}\right) /(n)<\hat{\ell}_{i}$. The former have increasing marginal utility in $\gamma$ and their peak is in $\gamma=1$. The others have decreasing marginal utility in $\gamma$ and their peak is in $\gamma=0$. Q.E.D.

Lemma 2 guarantees that there is an $\gamma$ that is a Condorcet winner (Black, 1958). Let us order agents in terms of their labor contributions. We denote the agent in the median by $m$. Agent $m$ is the one occupying the place $(n+1) /(2)$ in the order. The result of majority voting depends on the comparison of $\hat{\ell}_{m}$ and labor per capita.

Proposition 2. If $\hat{\ell}_{m}<\left(\sum_{i \in N} \hat{\ell}_{i}\right) /(n)$, the outcome of majority voting is the Equal Benefit Rule.If $\hat{\ell}_{m}>\left(\sum_{i \in N} \hat{\ell}_{i}\right) / n$, the outcome of majority voting is the Proportional Rule.

Proof. From Lemma 2 we can order agents according to the labor contribution in the unique Pareto efficient allocation. Then, the result follows. Q.E.D.

Proposition 2 suggests a polarization among the members of a cooperative. On the one hand those agents who are relatively 'hard working' would like rewards to be proportional to effort. On the other hand those agents who are relatively 'lazy' would favor equal benefit. It would be interesting to

\footnotetext{
${ }^{1}$ The constant returns to scale technology is not interesting any more because all the sharing rules considered in this section satisfy PCR.
} 
consider a model in which agents can move from a cooperative to another ('vote with their feet' as in the celebrated Tiebout, 1956 model). The formalization of this idea in a full-blown model is left for future research.

\section{Acknowledgements}

The authors are grateful to Jose Alcalde, Carmen Beviá, Iñigo Iturbe-Ormaetxe, Vincent Merlin, Jörg Naeve and Ignacio Ortuño-Ortín for comments on a preliminary draft. This research has been partially financed by CICYT PB 93-0940.

\section{References}

Black, D., 1958. The Theory of Committees and Elections, Cambridge University Press, Cambridge.

Corchón, L.C., Puy, M.S., 1997. Existence and Nash implementation of efficient sharing rules for a commonly owned technology, Mimeo, Universidad de Alicante.

Fleurbaey, M., Maniquet, F., 1996. Cooperative production: A comparison of welfare bounds. Games and Economic Behavior 17, 200-208.

Maniquet, F., 1996. Allocation rules for a commonly owned technology: The average cost lower bound. Journal of Economic Theory 69, 490-507.

Mas-Colell, A., 1980. Remarks on the game theoretic analysis of a simple distribution of surplus problem. International Journal of Game Theory 9, 125-140.

Moulin, H., 1987. Equal or proportional division of a surplus and other methods. International Journal of Game Theory 16, 161-186.

Moulin, H., 1990. Joint ownership of a convex technology: Comparison of three solutions. Review of Economic Studies 57, 439-452.

Moulin, H., Roemer, J.E., 1989. Public ownership of the external world and private ownership of self. Journal of Political Economy 97, 347-367.

Pfingsten, A., 1991. Surplus sharing methods. Mathematical Social Sciences 21, 287-301.

Roemer, J.E., 1996. Theories of Distributive Justice, Chap. 6, Harvard University Press, London, Cambridge, MA.

Roemer, J.E., Silvestre, J., 1989. Public ownership: Three proposals for resource allocation, Mimeo, University of California.

Roemer, J.E., Silvestre, J., 1993. The proportional solution for economies with both private and public ownership. Journal of Economic Theory 59 (2), 426-444.

Tiebout, C.M., 1956. A pure theory of local expenditure. Journal of Political Economy 64, 416-424. 